Original Research Article

\title{
A study on knowledge, awareness and preventive practice about tuberculosis among medical students in Udaipur, India
}

\author{
Balaji D. More ${ }^{1}$, Chintan Doshi ${ }^{1}$, Vijay Baghel ${ }^{1}$, Anju B. More ${ }^{2}$
}

${ }^{1}$ Department of Pharmacology,

${ }^{2}$ Department of Anatomy, Pacific Institute of Medical Sciences, Udaipur, Rajasthan, India

Received: 07 October 2019

Revised: 14 November 2019

Accepted: 15 November 2019

\section{*Correspondence to:}

Dr. Balaji D. More,

Email: drbdmore@gmail.com

Copyright: (C) the author(s), publisher and licensee Medip Academy. This is an openaccess article distributed under the terms of the Creative Commons Attribution NonCommercial License, which permits unrestricted noncommercial use, distribution, and reproduction in any medium, provided the original work is properly cited.

\begin{abstract}
Background: Tuberculosis (TB) is a highly lethal infectious disease causing huge health burden worldwide, commonly involving the respiratory system. Medical students should also be empowered to impart awareness and reduce stigma related to TB. The objective of this study was to evaluate whether educational intervention would affect the level of TB awareness, knowledge, attitude and preventive practice of TB among medical students.
\end{abstract}

Methods: The present study was carried out among the second year medical college students of Pacific Institute of Medical Sciences, Udaipur, India $(n=142)$. A multiple-choice questionnaire of 45 questions was designed in English language, for pre- and post-tests. A 30-minute visual health education was given on TB and the assessment of knowledge, attitude and preventive practice of TB was done by a pre-test and post-test questionnaire.

Results: Out of the total 150 student, 142 participated in the study making the response rate $94.66 \%$. The total mean percentage of correct answers for TB knowledge was $48.59( \pm 20.44)$ which improved to $69.33( \pm 17.74)$ post-training. The total mean percentage of right attitude was $63.036( \pm 16.46)$ which improved to $77.06( \pm 15.58)$ post-training. The total mean percentage of correct answer for preventive practice were provided by $67.036( \pm 12.90)$ which changed to $79.50( \pm 12.01)$ post-training. There was significant difference in the knowledge, attitude and preventive practice of students in pre- and post-training tests $(\mathrm{p}=0.001)$.

Conclusions: Our findings revealed that medical students had poor knowledge. A simple TB education session had a positive influence on knowledge, attitude and preventive practices about TB among them.

Keywords: Attitude, Knowledge, Medical students, Practice, TB

\section{INTRODUCTION}

Worldwide, 9.7 million people are affected by tuberculosis (TB). ${ }^{1}$ TB remains to be a major public health burden across the globe, including India. India alone accounts for approximately $24 \%$ of all incident TB cases globally. ${ }^{2}$ According to a recent WHO report approximately one million deaths occur due to TB every year globally, of which 0.24 million were Indians. ${ }^{2}$ It is not only a cause of ill-health but ranks second as the leading cause of death from an infectious disease. ${ }^{3}$ TB has high morbidity and mortality, one new infected case per second and one death every 10 seconds. ${ }^{4}$ Increasing the awareness in the medical students leads to early diagnosis of TB which is one of the mainstays strategy to eradicate TB. Sufficient knowledge and a positive outlook towards TB patients among medical student and nurses are required to achieve the Sustainable Development Goals proposed by the WHO as a part of its post-2015 global TB strategy and to successfully combat the newer challenges posed by this disease in the future.

Furthermore medical students play a valuable public health role in promoting community awareness of TB particularly in reducing stigma attached to TB. Studies in southern parts of India have shown that there is need for addressing the gaps regarding the knowledge and transmission of $\mathrm{TB}$ among the medical students and 
professionals. Though few studies were conducted on knowledge, attitude and preventive practice (KAP) towards TB in India, no study has been done about KAP towards TB among medical student in our Institute. Therefore, this study has been designed to investigate KAP of medical and nursing student towards TB.

\section{METHODS}

The study design was a prospective, survey, with intervention of training. This study was conducted at Pacific Institute of Medical Sciences, Udaipur, India between August 2019 to September 2019. It includes a self-administered pre-tested questionnaire was completed by medical students. The students included in this study were second year undergraduates willing to participate in this study. The institutional ethics committee approval was obtained for this study. The A questionnaire was designed consisting of 45 questions, of which 20 were on knowledge, 14 on attitude and 11 on the preventive practice with multiple-choice answers. A 30-minute visual health education was given on TB in English. The assessment was carried out with the help of a pre-test and post-test. The students were instructed to encircle the most appropriate answer independently without receiving any assistance to fill in the questionnaire. Their participation was on a voluntary basis and they were not provided any remuneration.

The data were collected related to awareness on general aspects of $\mathrm{TB}$, its prevention and treatment and also the attitude of these students towards TB. The data obtained were recorded in a Microsoft Excel sheet, and statistical analysis was performed using statistical package for the social sciences (version 17). Results are presented as drawings, frequency and percentages. Categorical variables were compared using Mc Nemer's Chi-square test. Quantitative results were compared with paired t test and Wilcoxon signed rank test. For all tests, significant was achieved at $\mathrm{p}<0.05$. All performed statistical tests were two tailed.

\section{RESULTS}

Out of the total 150 student, 142 participated in the study making the response rate 94.66. The total mean percentage of correct answers for TB knowledge was $48.59( \pm 20.44)$ which improved to $69.33( \pm 17.74)$ in post training session $(\mathrm{p}=0.001)$. The total mean percentage of right attitude was $63.036( \pm 16.46)$ which improved to $77.06( \pm 15.58)$ by post training session. The total mean percentage of correct answer for preventive practice were provided by $67.036( \pm 12.90)$ which changed to 79.50 $( \pm 12.01)$ during post training session. There was significant difference in the KAP among students during pre and post training tests $(\mathrm{p}=0.001)$. Our findings revealed poor knowledge, attitude and preventive practise among second year medical students regarding TB, which improved to moderate levels.

\section{Knowledge about TB}

The causative agent of TB was known to almost $80 \%$ of the respondent, and after training session the percentage increase to $91.5 \%$. About $60.6 \%$ of respondents were aware about the symptoms of TB such as cough $\geq 3$ weeks, loss of appetite, weight loss fever or chill, chest pain, and tiredness/fatigue. Post-training the corrects respondent percentage increased to $68.3 \%$. Almost $76.8 \%$ mentioned that droplet inhalation as the main mode of transmission of the disease. Post training $88 \%$ responded correctly. Only $31.7 \%$ respondents were knew about the screening test for latent TB infection. The training improved this percentage to $52.1 \%$.

Regarding sputum sample collection for pulmonary TB diagnosis, $40.8 \%$ mentioned 3 (spot, morning, spot) samples as most useful method of sputum sample collection. Post training 69.7 \%responded correctly for pulmonary TB diagnosis which was significantly higher.

Before training only $9.2 \%$ and $32.4 \%$ knew that isoniazid and kanamycin are first and second line anti-TB drugs respectively. After training about $63 \%$ participant knew the right answers $(\mathrm{p}<0.0001)$. Pre-training $57.0 \%$ students were aware of multidrug-resistant TB (MDRTB) and its causative bacteria resistant to isoniazid and rifampicin. During post-test, the percentage of students knowing this increased to $71.10 \% \quad(\mathrm{p}=0.02)$. Before training $35.2 \%$ recognized that extensively drug-resistant TB (XDR-TB) is resistant to any fluoroquinolone and at least one of the three injectable second-line drugs. The training improved this number to $42.96 \%$. Sixty-five $(45.8 \%)$ students identified the duration of standard treatment for new patient with drug-sensitive TB as 4-12 months, which altered to $56.34 \%$ after training.

Pre-training bout $77.5 \%$ student stated that HIV patients are more vulnerable to contracting TB, while posttraining the percentage increased to $85.9 \%$. About 73.2 study participants thought that $\mathrm{TB}$ is a curable disease, the awareness increased to $86.6 \%$ post-training. More than half (53) study participants stated that MDR-TB is a curable disease, that changed to 90 post-training. An airborne mode of transmission of pulmonary TB was stated by $68 \%$ students which changed to $83.8 \%$. Very few students $(38.7 \%)$ knew that patient with latent TB do not spread the disease, the awareness increased to $85.9 \%$ post-training. Patient with TB usually become noninfectious soon after initiating appropriate treatment and only patient with active TB can spread the disease; which was known to $26.1 \%$ and $38.7 \%$ and the awareness increased to $41.0 \%$ and 79.5 post-training respectively.

About $70 \%$ students were aware about the usage of personal protective equipment (PPE) when dealing with a patient with active pulmonary $\mathrm{TB}$ and awareness increased to $83.1 \%$ post training. 


\section{Attitude towards TB}

Regarding attitude towards TB, the number of students willing to work in TB clinic or ward increased from 73 to 105 but even after training, 122 of 142 students declined actual posting. The students' awareness on need for their own screening in presence of suggestive symptoms improved to $88.7 \%$. Study participants willingness to share knowledge with patients and co-workers improved to $93 \%$ that included accurate method of sputum collection. Almost $75 \%$ were willing to teach patient how to collect sputum sample. Majority $(91.6 \%)$ of student agreed to trust the result the laboratory provides you on sputum cultures.

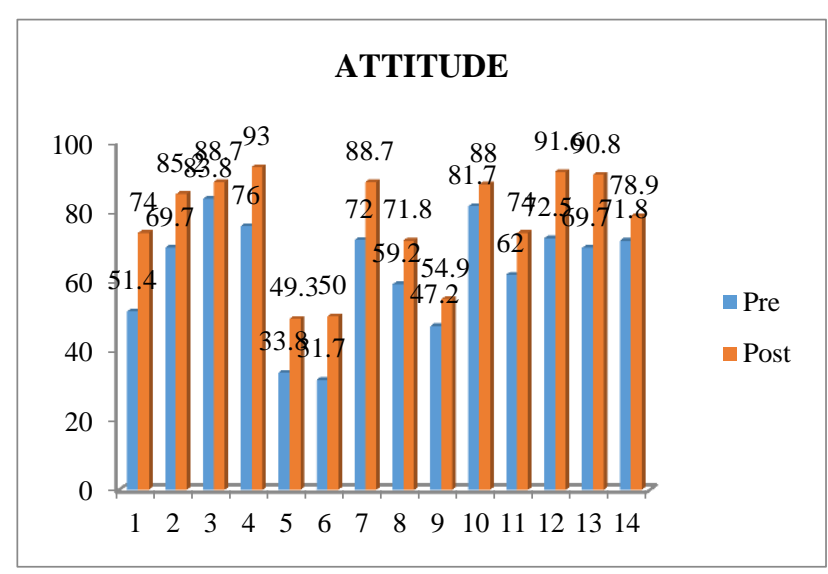

Figure 1: The percentage of responses with positive attitude in pre- and post-test assessment.

1: Would you be willing to work in a TB clinic/ward?; 2 : Would you resign from work if you are posted to a TB clinic/ward?; 3: Would you be willing to be screened for TB if you had suggestive symptoms?; 4: Would you be willing to teach patients and co-workers about TB prevention; 5: Do you think all TB patients should be isolated for treatment?; 6: It is ok to allow a TB patient to leave the hospital soon after initiating appropriate treatment?; 7: Would you be willing to attend seminars on TB?; 8: Would you recommend the suspension of treatment if a TB patient is feeling better?; 9: Would you start TB treatment for a TB patient before diagnosis is confirmed if a suspected TB patient is very ill; 10: Would you use a face mask when dealing with a pulmonary TB patient even when it is uncomfortable?; 11: Would you be willing to teach patient how to collect sputum sample?; 12: Would you trust the result the laboratory provides you on sputum cultures?; 13: I would accept to examine/treat a TB patients; 14: I worry about acquiring active TB disease while at work.

The students improved on their view about need for isolation and hospitalisation and continued therapy after initial improvement in TB patients. They also had better inclination to treat and update their knowledge through seminars followed by training. Though, with the training they had more worry regarding acquiring TB the utility of simple face mask when dealing with a pulmonary TB patient was more evident even when it is uncomfortable.

\section{Practice towards prevention of $\mathrm{TB}$}

Students $(95 \%)$ agreed to perform hand hygiene and wear PPE before contact with pulmonary TB patient or TB samples. They $(82 \%)$ would wear N95 respirator when caring for patient with pulmonary TB or working on TB samples. Most of them $(91.5 \%)$ would request sputum tests when they suspect active TB. More than $73 \%$ would admit active TB patients in isolation wards with better natural ventilation. About $80 \%$ realised the significance of HIV test in TB cases. About $61.3 \%$ of student may not use wet or soiled N95 respirator. They (88 \%) would always make sure that samples are sputum and not saliva before sending samples to laboratory. The necessity of contact tracing is now confirmed by $83.1 \%$ as opposed to $68.3 \%$ prior to training.

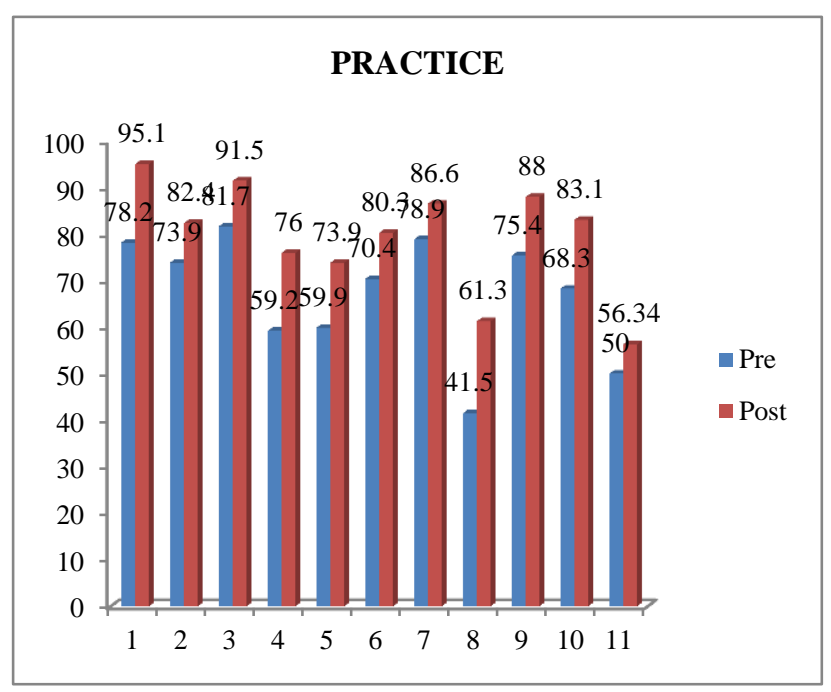

\section{Figure 2: The percentage of correct responses about practice in pre- and post-test assessment.}

1: I usually perform hand hygiene and wear PPE before contact with pulmonary TB patient/TB samples; 2: Usually wear N95 respirator when caring for patient with pulmonary $\mathrm{TB} /$ working on $\mathrm{TB}$ samples; 3: I request sputum tests when I suspect active TB; 4: Always put the patient with active $\mathrm{TB}$ in an isolated room; 5: I open windows when possible in TB patients rooms to increase natural ventilation; 6: I order HIV test when I diagnose active TB; 7: Always put the patient with known TB separated from HIV patients; 8: Awareness to avoid use of wet or soiled N95 respirator; 9: I always make sure that samples are sputum and not saliva before sending them to; 10: I request contact tracing for all confirmed TB cases; 11: I request liver function tests before starting anti-TB treatment. 
Table 1: The total mean percentage of correct knowledge, positive attitude and correct practice in pre- and posttest assessment.

\begin{tabular}{|c|c|c|c|c|c|c|c|}
\hline \multirow{2}{*}{ Mean \% of } & \multicolumn{2}{|l|}{ Pre-test } & \multicolumn{2}{|c|}{ Post-test } & \multirow{2}{*}{$\begin{array}{l}\text { Mean } \\
\text { difference }\end{array}$} & \multirow{2}{*}{$\begin{array}{l}\text { Paired } t \text { test/Wilcoxon } \\
\text { signed rank test }\end{array}$} & \multirow{2}{*}{ P value } \\
\hline & Mean & $\pm \mathrm{SD}$ & Mean & $\pm \mathrm{SD}$ & & & \\
\hline Knowledge & 48.59 & 20.44 & 69.33 & 17.74 & 20.74 & $t=6.438$ & $0.001 *$ \\
\hline Attitude & 63.036 & 16.46 & 77.06 & 15.58 & 14.04 & $\mathrm{z}=3.297$ & $0.001 *$ \\
\hline Practice & 67.0364 & 12.90366 & 79.5036 & 12.01360 & 12.47 & $t=9.493$ & $0.001 *$ \\
\hline
\end{tabular}

*: Significant difference.

\section{DISCUSSION}

TB risk is increased among health care workers (HCW) in low- and middle-income countries (LMIC) in comparison to the general population. ${ }^{5-7}$ Moreover, TB disease risk is particularly high among new medical students. In a retrospective study, during 2012-2013, at a public medical institute at Pune, India, 26 (3.9\%) cases of TB were found amid 662 medical students, signifying a projected incidence of 3,279 cases/100,000 person-years. Out of which 3 cases were isoniazid-resistant, 1 was multidrug-resistant, and 1 case had fulminant hepatitis post TB treatment. ${ }^{8}$ Medical students possibly have increased risk because of increased duration of exposure to TB patients in the hospital. Another study from India stated a 4-fold higher prevalence of TB infection among older medical students (i.e., >23 years of age) compared with younger medical students (18-20 years of age). ${ }^{9}$ Although occupational TB exposure has gained increased attention and awareness, latent TB infection (LTBI), an indicator of TB exposure, remains poorly characterized in high TB burden settings. It becomes imperative to take measures to prevent infection in this vulnerable groups of individuals.

Increasing the knowledge, improving the attitude and preventive practice is one way of protecting this student. Past studies also have emphasized the immediate need for education and implementation of infection control measures to safeguard medical students from TB. ${ }^{8,10}$ Medical students still are in need of continuing educational programs regarding treatment and control of TB. Thus, creating awareness at student and community level could play a vital role in control and prevention of TB. ${ }^{11}$ Moreover knowledge about TB will also help them to take proper precaution during patients care. ${ }^{12}$ Inadequate TB knowledge was observed among Chinese medical students, which indicates a requirement to transform our current infectious disease curriculum to increase TB knowledge and preventive practices among medical students. ${ }^{13}$

The National Institutes of Health funded the National Tuberculosis Curriculum Consortium (NTCC) to enhance skills and inculcate correct attitudes in the management and control of TB among medical students in their formative years. ${ }^{14}$ Numerous studies have assessed the knowledge and attitudes of medical students about TB. The study by Emili et al. demonstrated significant differences in student knowledge and practice competency in regards to TB at 3 medical schools. ${ }^{15} \mathrm{~A}$ study in Istanbul Medical showed that the TB-related examination questions did not adequately reflect WHO learning objectives. Also the students' skills developed with practical training on $\mathrm{TB}$ were insufficient. ${ }^{16}$ In another study, a scope for improvement in a baseline level of knowledge, attitudes, and confidence about TB was discussed. ${ }^{17}$

A recent study evidently showed that a simple, 30-minute training had a positive impact on knowledge and awareness about TB among school children. An increase in mean knowledge score from pre-test to post-test, indicate that empowerment of students could guide the community on various aspects of TB. ${ }^{11}$ Studies have documented a positive association between TB knowledge, care seeking and treatment adherence. ${ }^{18,19}$ To address such issues, the level of knowledge should be known to design an appropriate interventional programs in a specific regions. ${ }^{20}$ Inadequate TB knowledge was observed among Chinese medical students, which indicates a requirement to transform our current infectious disease curriculum to increase TB knowledge and preventive practices among medical students. ${ }^{13}$ Improved knowledge on $\mathrm{TB}$ will thus contribute to effective TB control and in the long term, will help India achieve the goal of End TB. ${ }^{21}$

Worldwide prevalence of LTBI among HCWs is estimated at $54 \%$ with yearly risk reaching from $0.5 \%$ to $14 \% .{ }^{22-24}$ In an Indian study, high risk for LTBI was observed among medical students as compared to nursing students. Similar finding was reported in other LMIC This indicate high TB disease exposure in the hospitals and low awareness of and control over exposure to risk factors among medical students. ${ }^{25,26}$ WHO has led guidelines for infection control dissemination of knowledge, change in attitude and preventive practices to protect the HCWs at highest risk for LTBI. ${ }^{24,27}$

This study provided insights on the knowledge, selfreported attitudes and preventive practices among the medical student towards TB. Our pre-test results reveal significant gaps in all three areas which were significantly reduced by well-structured training as evident by the post-training scores.

It is a recognized fact that knowledge can influence people's practices regarding prevention. The current 
study revealed that the overall knowledge about TB was more than $50 \%$ similar to $62 \%$ among Iranian students. ${ }^{28}$ Our results were closure to the results reported by Laurenti, the total mean percentage of correct answers in our study was lower $(48.59 \%$ vs. $56.5 \%) .{ }^{29}$ Whereas the current finding was better than a study done in Thailand in which $74.2 \%$ respondents had low level of knowledge about $\mathrm{TB} .{ }^{30}$ In our study the percentage of corrects answers significantly improved post training.

Moreover, the general knowledge level for TB in our study was inferior to other surveys conducted among medical students in Italy, Brazil, and NTCC schools. ${ }^{31-33}$ This indicate a substantial necessity for enhancement in TB knowledge among medical students which may influence their pervasiveness in acquiring TB. The current study participant's overall correct attitude towards TB was $63.03 \%$ which is better than their Thailand counterparts, $47.9 \% .^{30}$ Further, in our study post-training the attitude improved in $77.06 \%$ students.

The finding from various studies indicate that treatment delay may be affected by several factors, such inadequate knowledge, lack of awareness of the significance of symptoms, negative social attitudes or combinations of these. ${ }^{34}$ Our findings suggest that the overall preventive practice towards TB was $67.03 \%$ among the students which were better than a study done in Iran in which the overall preventive practices towards TB was $42.6 \% .^{28}$ Whereas a study done in Thailand showed that $55.5 \%$ of study participants had high level of preventive behaviour. ${ }^{30}$ These difference are because the participant of our study are medical student who are more aligned toward medical education as a part of their career where those study participants from different countries may have differences in health education program design and health knowledge. Though, their poor knowledge about TB was evident with $>50 \%$ incorrect answers in present study as well as in southwest China study conducted in $2011 .^{35}$

The attitude towards TB significant affect how a doctor manage TB patients during their careers. ${ }^{36}$ In our study and Bhandari et al, majority of medical students lacked correct TB attitude due to fear of acquiring TB themselves. ${ }^{37}$ This prejudice was consistent with poor knowledge and preventive practices. Thus imparting knowledge can improve attitude towards TB but a higher levels of knowledge does not guarantee positive attitude. Essentially, correct knowledge and positive attitude of doctor can ensure better service to community.

Our results are also similar to the study conducted by Teixeira et al in Brazil. The commonalities were that many medical students were not aware of the main routes of TB infection and that training on TB was not sufficient to improve knowledge and practices. Irrespective of knowledge about TB transmission, students may engaged in risky behaviours as many of them may not a protective mask when examining an active TB case. The finding from various studies indicate that treatment delay may be influenced by several factors, namely lack of knowledge, lack of awareness of the significance of symptoms, negative social attitudes or combinations of these. ${ }^{18,38}$

Our findings are also in congruence with of several other studies among health professions students. Kilicaslan et al assessed the impact of TB training on medical students at Istanbul Medical School. They found many fourth-year medical students answered incorrectly. ${ }^{16}$ In Japanese study it was found that additional training on TD was required for doctors and medical students. ${ }^{39}$ Bai et al conducted a survey in final year medical students in China and had comparable findings. ${ }^{40}$ On the contrary study conducted by Emili et al in final year medical students from Canada, India, and Uganda found that although there were significant differences in undergraduate exposure to $\mathrm{TB}$, total knowledge, and practice competency among these students, the TB knowledge base and practice competency was satisfactory. ${ }^{15}$

To properly address the serious problems of TB prevention and control, education and competence should to be reinforced among medical students. International educational programmes have been developed to enhance TB knowledge and practices among medical students. ${ }^{33}$ Newer and modern training methods, such as studentbased teaching methods (problem-based learning) should be implemented enhance student satisfaction and offer extra learning opportunities.

Our study has several limitations including comprehensive and appropriate questionnaire adapted to Indian scenario, cross-sectional small sample size without randomization. However, the findings provide a basis for acquiring and testing a causal hypothesis. The study findings revealed that a simple TB education session for second year medical students can positively affect KAP about TB. Basic education on infectious diseases need further boost in medical curriculum and other educational programmes to improve social accountability.

Our findings revealed that second year medical students had poor knowledge which improved to moderate levels. Empowerment of students with knowledge could be helpful in spreading awareness on various aspects of TB and also protects them from the risk of getting infected during their training period in the hospital. This also urges the medical educational institute to provide basic education on infectious diseases like TB and design strategies to enhance KAP in the early years of medical curriculum. Our study results underscore the necessity to develop educational programmes and learning strategies to enhance knowledge and ensure social accountability of medical colleges.

\section{ACKNOWLEDGEMENTS}

We acknowledge study participants for their enthusiasm and our institution for infrastructural support. 
Funding: No funding sources

Conflict of interest: None declared

Ethical approval: The study was approved by the Institutional Ethics Committee

\section{REFERENCES}

1. Kasa AS, Minibel A, Bantie GM. Knowledge, attitude and preventive practice towards tuberculosis among clients visiting public health facilities. BMC Res Notes. 2019;12(1):276.

2. Uppada DR, Selvam S, Jesuraj N, Lau EL, Doherty TM, Grewal HMS, et al. Incidence of tuberculosis among school-going adolescents in South India. BMC Public Health. 2016;16:641.

3. Hibstu D, Bago B. Knowledge, attitude and practice of tuberculosis and its transmission among high school students in Yirgacheffe town, Gedeo zone, southern Ethiopia. J Infect Dis Preve Med. 2016;4(1000142):2.

4. Lönnroth K, Raviglione M. Global epidemiology of tuberculosis: prospects for control. Semin Respir Crit Care Med. 2008;29(5):481-91.

5. Joshi R, Reingold AL, Menzies D, Pai M. Tuberculosis among health-care workers in low-and middle-income countries: a systematic review. PLoS Medicine. 2006;3(12):e494.

6. Uden L, Barber E, Ford N, Cooke GS. Risk of tuberculosis infection and disease for health care workers: an updated meta-analysis. Open Forum Infect Dis. 2017;4(3):ofx137.

7. de Vries G, Šebek MMGG, Lambregts-van Weezenbeek CSB. Healthcare workers with tuberculosis infected during work. European Respiratory Journal. 2006;28(6):1216-21.

8. Basavaraj A, Chandanwale A, Patil A, Kadam D, Joshi S, Gupte N, et al. Tuberculosis Risk among Medical Trainees, Pune, India. Emerg Infect Dis. 2016;22(3):541-3.

9. Pai M, Gokhale K, Joshi R, Dogra S, Kalantri S, Mendiratta DK, et al. Mycobacterium tuberculosis infection in health care workers in rural India: comparison of a whole-blood interferon $\gamma$ assay with tuberculin skin testing. JAMA. 2005;293(22):274655.

10. Canetti G, Froman S, Grosset JA, Hauduroy P, Langerova $\mathrm{M}$, Mahler $\mathrm{H}$, et al. Mycobacteria: laboratory methods for testing drug sensitivity and resistance. Bull World Health Organ. 1963;29(5):565.

11. Vanaja K, Banu R, Reddy L, Kumar PC, Srinivas C, Rajani T, et al. A study on knowledge and awareness about tuberculosis in senior school children in Bangalore, India. Indian J Tuberc. 2016;63(3):192-8.

12. Acharya PR, D'Souza M, Sahoo RC. Tuberculosis knowledge and attitude in aspiring doctors and nurses - Is it time for our TB teaching methods to evolve? Indian J TB. 2017;64(1):20-5.

13. Ou Y, Luo Z, Mou J, Ming H, Wang X, Yan S, et al. Knowledge and determinants regarding tuberculosis among medical students in Hunan, China: a crosssectional study. BMC Public Health. 2018;18(1):730.

14. Harrity S, Jackson M, Hoffman H, Catanzaro A. The National Tuberculosis Curriculum Consortium: a model of multi-disciplinary educational collaboration. Int $\mathrm{J}$ Tuberc Lung Dis. 2007;11(3):270-4

15. Emili J, Norman GR, Upshur RE, Scott F, John KR, Schmuck ML. Knowledge and practices regarding tuberculosis: a survey of final-year medical students from Canada, India and Uganda. Med Educ. 2001;35(6):530-6.

16. Kilicaslan Z, Kiyan E, Erkan F, Gurgan M, Aydemir $\mathrm{N}$, Arseven O. Evaluation of undergraduate training on tuberculosis at Istanbul Medical School. Int J Tuberc Lung Dis. 2003;7(2):159-64.

17. Jackson M, Harrity S, Hoffman H, Catanzaro A. A survey of health professions students for knowledge, attitudes, and confidence about tuberculosis, 2005. BMC Public Health. 2007;7:219.

18. Storla DG, Yimer S, Bjune GA. A systematic review of delay in the diagnosis and treatment of tuberculosis. BMC Public Health. 2008;8(1):15.

19. Cramm JM, Finkenflügel HJ, Møller V, Nieboer AP. TB treatment initiation and adherence in a South African community influenced more by perceptions than by knowledge of tuberculosis. BMC Public Health. 2010;10(1):72.

20. Adane K, Spigt M, Johanna L, Noortje D, Abera SF, Dinant GJ. Tuberculosis knowledge, attitudes, and practices among northern Ethiopian prisoners: Implications for TB control efforts. PLoS One. 2017;12(3):e0174692.

21. Krithika SA, Jayanthi NN, Subramanian S. Awareness of tuberculosis among nurses. IAIM. 2018;5(4):153-60.

22. Ochoa J, Hincapie-Palacio D, Sepulveda H, Ruiz D, Molina A, Echeverri S, et al. Simulation of risk of tuberculosis infection in healthcare workers in hospitals of an intermediate incidence country. Epidemiol Infect. 2015;143(12):2639-47.

23. Rafiza S, Rampal KG, Tahir A. Prevalence and risk factors of latent tuberculosis infection among health care workers in Malaysia. BMC Infect Dis. 2011;11(1):19.

24. Kinikar A, Chandanwale A, Kadam D, Joshi S, Basavaraj A, Pardeshi G, et al. High risk for latent tuberculosis infection among medical residents and nursing students in India. PLoS One. 2019;14(7):e0219131.

25. Soto A, Huablocho P, Ulloque J. Early conversion of tuberculin skin test in medical students who begin hospital practices. Brazil J Infect Dis. 2013;17(3):383-4.

26. Teixeira EG, Menzies D, Comstock G, Cunha A, Kritski A, Soares L, et al. Latent tuberculosis infection among undergraduate medical students in Rio de Janeiro State, Brazil. Intl J Tuberculosis Lung Dis. 2005;9(8):841-7. 
27. Harries AD, Hargreaves NJ, Gausi F, Kwanjana JH, Salaniponi FM. Preventing tuberculosis among health workers in Malawi. Bull World Health Organ. 2002;80(7):526-31.

28. Bagheri Amiri F, Doosti-Irani A, Sedaghat A, Fahimfar N, Mostafavi E. Knowledge, Attitude, and Practices Regarding HIV and TB Among Homeless People in Tehran, Iran. Int J Health Policy Manag. 2018;7(6):549-55.

29. Laurenti P, Federico B, Raponi M, Furia G, Ricciardi W, Damiani G. Knowledge, experiences, and attitudes of medical students in Rome about tuberculosis. Medical Sci Monitor: Int Med J Exp Clin Res. 2013;19:865.

30. Sreechat S. Assessment of knowledge attitude and preventive behavior of pulmonary tuberculosis among Myanmar refugees in Ban Mai Nai Soi temporary shelter Mae Hong Son Thailand: Chulalongkorn University; 2012.

31. Montagna MT, Napoli C, Tafuri S, Agodi A, Auxilia F, Casini B, et al. Knowledge about tuberculosis among undergraduate health care students in 15 Italian universities: a cross-sectional study. BMC Public Health. 2014;14(1):970.

32. Teixeira EG, Menzies D, Cunha AJLA, Luiz RR, Ruffino-Netto A, Scartozzoni MS, et al. Knowledge and practices of medical students to prevent tuberculosis transmission in Rio de Janeiro, Brazil. Revista Panamericana de Salud Pública. 2008;24:265-70.

33. Jackson M, Harrity S, Hoffman H, Catanzaro A. A survey of health professions students for knowledge, attitudes, and confidence about tuberculosis, 2005. BMC Public Health. 2007;7(1):219.
34. Koay T. Knowledge and attitudes towards tuberculosis among the people living in Kudat District, Sabah. Med J Malaysia. 2004;59(4):502-11.

35. Zhao Y, Ehiri J, Li D, Luo X, Li Y. A survey of TB knowledge among medical students in Southwest China: is the information reaching the target? BMJ Open. 2013;3(9):e003454.

36. Emili J, Scott F, Upshur R, Schmuck M, John K. Attitudes toward tuberculosis of final year medical students from Canada, India, and Uganda. Teaching Learn Med. 2002;14(3):168-74.

37. Bhandari SR, Bande R. Knowledge, attitude and practice against tuberculosis infection control among medical students and nursing staff. J Cont Med A Dent. 2016;4(2):29-32.

38. Seid A, Metaferia Y. Factors associated with treatment delay among newly diagnosed tuberculosis patients in Dessie city and surroundings, Northern Central Ethiopia: a cross-sectional study. BMC Public Health. 2018;18(1):931.

39. Kurane $\mathrm{S}$, Kudoh S. The importance of the examination of, education on, and infection control of tuberculosis in medical school hospitals in Japan. Kekkaku (Tuberculosis). 2003;78(9):573-80.

40. Bai L, Xiao S, Xie H, Yang G, Wang Y. Knowledge and practice regarding tuberculosis among final-year medical students in Hunan, China. Zhonghua jie he he hu xi za zhi= Zhonghua jiehe he huxi zazhi= Chin J Tuberculosis Respirator Dis. 2003;26(8):458-61.

Cite this article as: More BD, Doshi C, Baghel V, More AB. A study on knowledge, awareness and preventive practice about tuberculosis among medical students in Udaipur, India. Int J Basic Clin Pharmacol 2019;8:2706-12. 